\title{
Design and Implementation of the Intelligent Convergence Security System for Hazard Event on IoT Environments
}

\author{
Junho Jeong ${ }^{1}$, Dong Ha Park ${ }^{2}$, Jun Young Lee ${ }^{2}$, Uduakobong George Offong ${ }^{2}$, \\ Seman $\mathrm{Oh}^{2}$ and Yunsik Son ${ }^{2 *}$ \\ ${ }^{1}$ Electrocnic Commerce Institute, Dongguk University \\ ${ }^{2}$ Department of Computer Science and Engineering, Dongguk University \\ \{yanyenli, poongbeck, kuroneko0441, 2017120224, smoh, sonbug\}@dongguk.edu
}

\begin{abstract}
Security refers to the protection of tangible and intangible assets such as individuals, countries, companies, etc. Security can be largely classified into Information Security and Physical Security. Information security refers to security products and services to prevent damage, modification, and leakage of information on a computer or network. Physical security refers to security products and services for the safe operation of major facilities and for preventing disasters, and crimes. The need for security convergence is increasing as traditional physical security becomes hardware-centric and requires areas of software such as image analysis, data collection and management. For this reason, many researches on security convergence applying IoT technology to traditional physical security have been underway due to recent developments of various sensors and internet environments. In this paper, we have developed an event-based approach to risky and limited facilities access and user anomalies. We propose an intelligent security convergence model based on IoT environment event for integrated tasks.
\end{abstract}

Keywords: Convergence Security, Internet of Things, Multi-Sensors, Hazard Event

\section{Introduction}

Security refers to the safe-guarding of tangible and intangible assets such as individuals, countries, enterprises, etc. Security is largely categorized into information security and physical security. Information security is the securing of products and services from damage, tampering, or leakage of information on a computer or network. Physical security refers to the securing of products and services for the safe operation of major facilities and for preventing disasters, and crimes.

Security detection using CCTV, DVR, etc., are used in traditional physical security. As security detection and controller area have switched from analog based to IP based, the need for integrated control of collected data is also increasing [1-5].

Security requirements have moved beyond fixed spaces such as buildings, offices, and homes to mobile entities. The need for security convergence is increasing as more hardware-centric security require areas of software such as image analysis, data collection and management.

For this reason, many researches on security convergence applying IoT technology to traditional physical security, have been carried out [6-10]. As shown in Table 1, security convergence services are required in various industries. In the manufacturing industry, it is used to identify gas \& chemical levels, the work access history and status of local workers. In the construction industry, it is used to monitor the status of workers working on highrise buildings, real-time management of workforce, and manpower management.

Received (January 8, 2018), Review Result (March 9, 2018), Accepted (March 12, 2018)

* Corresponding Author 
Major security facilities such as nuclear power plants and substations require security convergence for the management of workers working in dangerous areas. Security convergence can estimate the state of mind and understand the current behavior of workers based on their activities, location, or heart rates.

In this paper, we propose an intelligent convergence security model based on IoT environment event to integrate hazard and limited facility access and user anomalies. In Section 2, we introduce details of the proposed convergence security model. We analyze the experiment in Section 3 and conclude in Section 4.

Table 1. The Need and Solution of Physical Security Service by Industry

\begin{tabular}{|c|c|c|}
\hline Industry & Need & Solution \\
\hline Manufacturing & $\begin{array}{l}\text {-Gas \& Chemical local worker } \\
\text { location status } \\
\text {-Identification of worker access } \\
\text { history }\end{array}$ & $\begin{array}{l}\text {-Leak detection around } \\
\text { storage in handling } \\
\text { chemicals } \\
\text { - Real-time check of heart }\end{array}$ \\
\hline Construction & $\begin{array}{l}\text {-Monitor worker's situation at } \\
\text { high place } \\
\text {-Real-time management of } \\
\text { dangerous workplace workers }\end{array}$ & $\begin{array}{l}\text { beat of worker } \\
\text { - Identify the location of the } \\
\text { entire worker in the event of } \\
\text { a hazard } \\
\text { - Expected announcement of } \\
\text { dropping point }\end{array}$ \\
\hline Major Facilities & $\begin{array}{l}\text { - Identify worker in hazardous } \\
\text { areas such as nuclear power } \\
\text { plants and substations }\end{array}$ & $\begin{array}{l}\text {-Monitoring the leakage of } \\
\text { dangerous area } \\
\text { - Worker real-time tracking }\end{array}$ \\
\hline
\end{tabular}

\section{An Intelligent Convergence Security Platform}

Our proposed platform structure is shown in Figure 1. The sensors used are BLE, GPS, gyroscope, acceleration, and heart rate measurement sensors. The monitoring app performs the first data preprocessing based on the information collected from the sensor, and then transmits the preprocessed data to the driver.

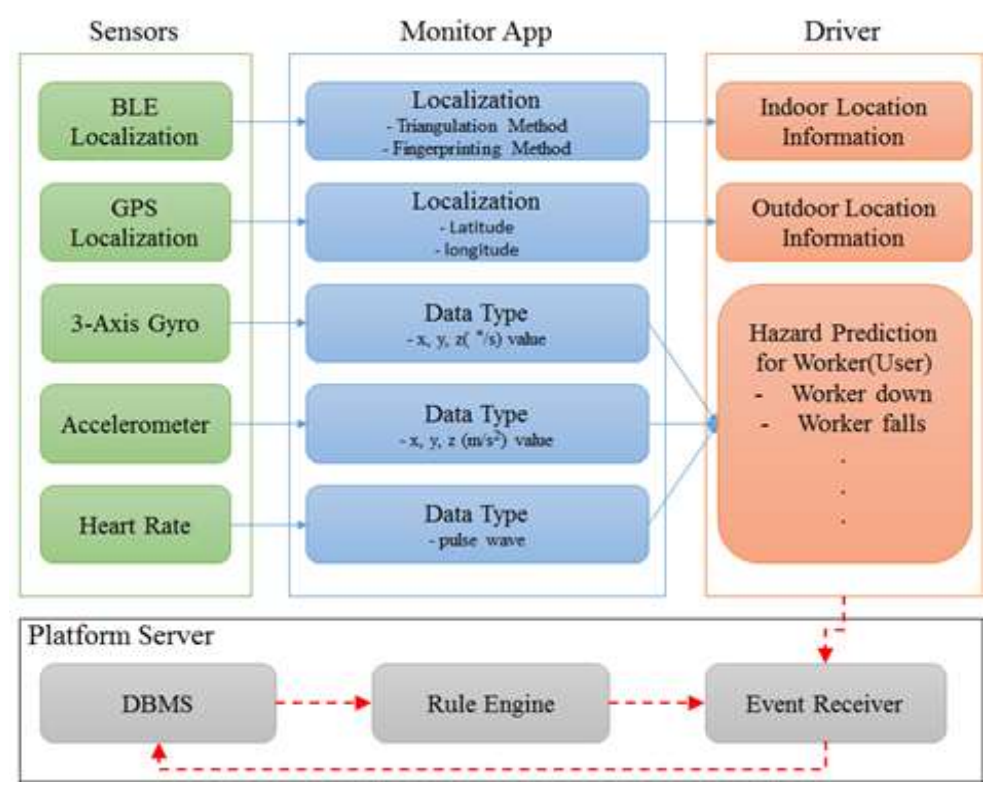

Figure 1. The Structure of an Intelligent Convergence Security Platform 
The driver can not only track the indoor / outdoor position of the user based on the information, but it can also be an event that predicts a dangerous situation with respect to the user's current state of mind based on information from the heart rate, acceleration and gyro sensors. The platform server receiving the event performs risk processing according to the event, records the content in the DBMS, and uses it as rules for improving the event processing performance in the future.

The proposed system judges and generates events based on the data received in real time through the relay driver without directly transmitting the data sensed by sensors to the DB.

\subsection{Communication Protocol for Monitoring App and Driver}

There is a need for a communication protocol for transmitting data collected from a plurality of sensors to the relay driver and performing connection management. Therefore, we designed and implemented a TCP / IP - based communication protocol capable of reliable data transmission. The definitions of the protocol packets are shown in Table 2.

Table 2. Definition of Proposed Communication Protocol Packet

\begin{tabular}{|c|c|c|c|c|}
\hline Struct & Field & Byte & Type & Note \\
\hline \multirow{7}{*}{ Prefix } & Target & 4 & Int & $\begin{array}{l}\text { What the sensor is monitoring. Use } 0 \\
\text { if there are no targets or unknowns }\end{array}$ \\
\hline & DeviceID & 4 & Int & Sensor/Event Identification number \\
\hline & Type & 4 & Int & Type of Event/Data \\
\hline & Length & 4 & Int & Byte Length of Data Area \\
\hline & Time & 8 & DateTime & Estimate Time by Sender \\
\hline & Reserved & 8 & Zero & Reserved Area \\
\hline & Total & 32 & & \\
\hline \multirow{13}{*}{$\begin{array}{l}\text { IMU Raw } \\
\text { Data }\end{array}$} & $\operatorname{Acc} X$ & 4 & Float & Linear Acceleration $\mathrm{X}$ axis \\
\hline & AccY & 4 & Float & Linear Acceleration $\mathrm{Y}$ axis \\
\hline & AccZ & 4 & Float & Linear Acceleration $\mathrm{Z}$ axis \\
\hline & padding & 4 & Zero & \\
\hline & GravX & 4 & Float & Gravity Acceleration $\mathrm{X}$ axis \\
\hline & GravY & 4 & Float & Gravity Acceleration $\mathrm{Y}$ axis \\
\hline & GravZ & 4 & Float & Gravity Acceleration $\mathrm{Z}$ axis \\
\hline & padding & 4 & Zero & \\
\hline & RotX & 4 & Float & Gyroscope $\mathrm{X}$ axis \\
\hline & RotY & 4 & Float & Gyroscope $\mathrm{Y}$ axis \\
\hline & RotZ & 4 & Float & Gyroscope $\mathrm{Z}$ axis \\
\hline & padding & 4 & Zero & \\
\hline & Total & 48 & & \\
\hline \multirow{4}{*}{$\begin{array}{l}\text { Zone } \\
\text { Data }\end{array}$} & BeaconID & 16 & UUID & $\begin{array}{l}\text { Bluetooth Low Energy Beacon } \\
\text { Identification Number }\end{array}$ \\
\hline & Major & 2 & Int & Major Number of Beacon \\
\hline & Minor & 2 & Int & Minor Number of Beacon \\
\hline & Total & 20 & & \\
\hline
\end{tabular}

Using TCP and Big Endian, the streaming processing of an Event / Raw Sample is possible without data loss. In order to support various kinds of data, it is divided into a prefix and a data area. Basically, event determination and generation are performed in the 
relay driver. The device is taken into consideration and reflected in the design. The process of collecting packets of devices using I / O DLLs, transmitting sensor values, and performing the DB query and using the relay driver to determine the event are as follows.

The Device sends its unique ID after the TCP socket connection. The relay driver checks the ID and sends 'Accept' when it is ready to receive data through the socket. The device then receives the current relay driver time (server time). Also, in the connection, the prefix is used without the use of the data area.

If explicitly terminated, the device sends a message to the relay driver to terminate the connection, and the relay driver that acknowledges the message terminates the connection.

\subsection{Database Design to Management Sensing Data}

The relay driver receiving the sensed data not only identifies and generates the event, but also stores the raw data in the database. Figure 2 is an overall table relationship diagram of a database designed to unify management of the sensed data for integrated control.

All devices that the relay driver allows to access must be registered in the DB in advance. The device table exists for this purpose. When the connection with the device is established, the relay driver updates the InTime (login time) and updates the OutTime (logout time) when the connection to the device is disconnected.

The event table is used to record the connection and disconnection of the devices. All devices must be registered prior to connection. The IDs used in this table are used for event recording. However, the codes and priorities of the events defined so far are defined but not used.

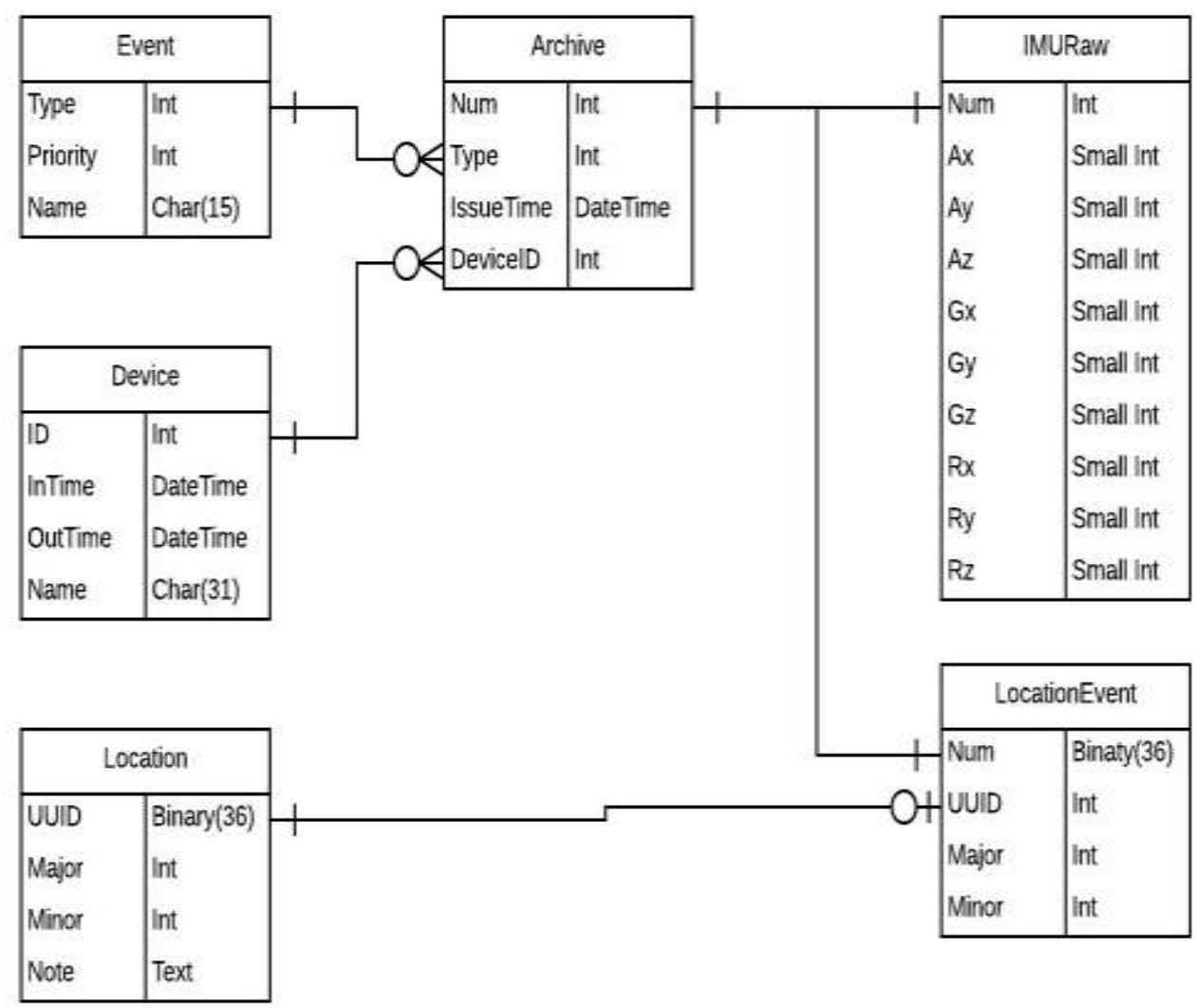

Figure 2. The Database Design of Proposed System 
All events are stored in the Archive table. An example of how events are stored is shown in Figure 3. Because it receives data from a plurality of devices, it can be confirmed that other device IDs are stored as values even if they have the same issue time.

Some events have additional data, for example, IMU Raw. IMU (acceleration + gyro) refers to sensor sampling data. The additional data records the number (Num) after the event is recorded by external reference.

\begin{tabular}{|c|c|c|c|}
\hline Num | & /pe & IssueTime & DeviceID | \\
\hline 1853 & 8 & 2017-08-30 08:30:31 & 17 \\
\hline 1863 & 8 & 2017-08-30 08:30:32 & 17 \\
\hline $1873 \mid$ & 8 & 2017-08-30 08:30:32 & 13 \\
\hline 1883 & 8 & 2017-08-30 08:30:32 & 17 \\
\hline 1893 & $8 \mid$ & 2017-08-30 08:30:32 & 13 \\
\hline 1903 & $8 \mid$ & 2017-08-30 08:30:32 & 17 \\
\hline 1913 & $8 \mid$ & 2017-08-30 08:30:32 & 13 \\
\hline 1923 & $8 \mid$ & 2017-08-30 08:30:32 & 17 \\
\hline 1933 & $8 \mid$ & 2017-08-30 08:30:32 & 13 \\
\hline 1042 & 8 & $2017-08-30$ 08:30:32 & 17 \\
\hline
\end{tabular}

Figure 3. Example of Storing Event in the Archive Table

\subsection{Hazard Event Generation Algorithm}

Various hazardous events will be supported by the proposed security platform. However, in this paper, we have designed an algorithm to generate events for occurrences where there is no motion after a significant fall. We designed the event generation algorithm using sudden changes of sensor acceleration attached to workers in the case of a sudden fall or lack of movement.

As a result of analyzing the actual sensed data, we found that, in the case of the workers who had fallen, the variation of acceleration increases instantaneously compared to general situations such as walking. As a result, we could implement the hazard event generation algorithm as shown in Figure 4.

$$
\begin{aligned}
& \text { if acc( } \mathrm{t})>\text { threshold }_{f} \\
& \text { list }=\operatorname{acc}(\text { from }(\mathrm{t}) \text { to }(\mathrm{t}+3 \mathrm{sec})) \\
& \text { if(list.max }- \text { list.min })>\text { threshold }_{f} \\
& \text { event }(\text { "fall") } \\
& \text { list=acc }(\text { from }(\mathrm{t}+3 \mathrm{sec}) \text { to }(\mathrm{t}+6 \mathrm{sec})) \\
& \text { if list.max }<\text { threshold }_{s f} \\
& \text { event("significant fall") }
\end{aligned}
$$

\section{Figure 4. Fall Hazard Event Generation Algorithm}

The algorithm returns the result of the events of fall and significant fall as input the minimum and maximum values of the acceleration within the last 3 seconds and the thresholds are threshold $_{f}$ and threshold $s$.

First, when a sudden change in acceleration occurs above a threshold $f$ a hazard event is prepared to generation. And a "Fall" event generates when the difference between the 
maximum acceleration and the minimum acceleration within 3 seconds exceeds the threshold $_{f}$. Also, if the maximum value of the acceleration is less than threshold $_{s f}$ within 3 seconds after the "Fall" event is generated, it is judged that there is no motion and a "Significant Fall" event is generated.

\section{Experiment}

We have experimented to confirm that we can generate real events based on the risk event generation algorithm proposed in the previous section. The experiment was based on an industrial scene, in which there is no action from an employee makes no after being hit by an external shock. The experiment takes 10 seconds and the experimenter will walk for 3 seconds and fall over on the prepared mat. After that, the experimenter does not move for 5 seconds. The whole experiment was conducted on 2 females and 1 male with average body size, and each experimenter fell down 20 times.

The results of the whole experiment show that the accelerations of all the experimenters were kept within $5 \mathrm{~m} / \mathrm{s}^{2}$ when walking before falling down, and the acceleration changed suddenly when the participants fell on the floor after 3 seconds from the start.

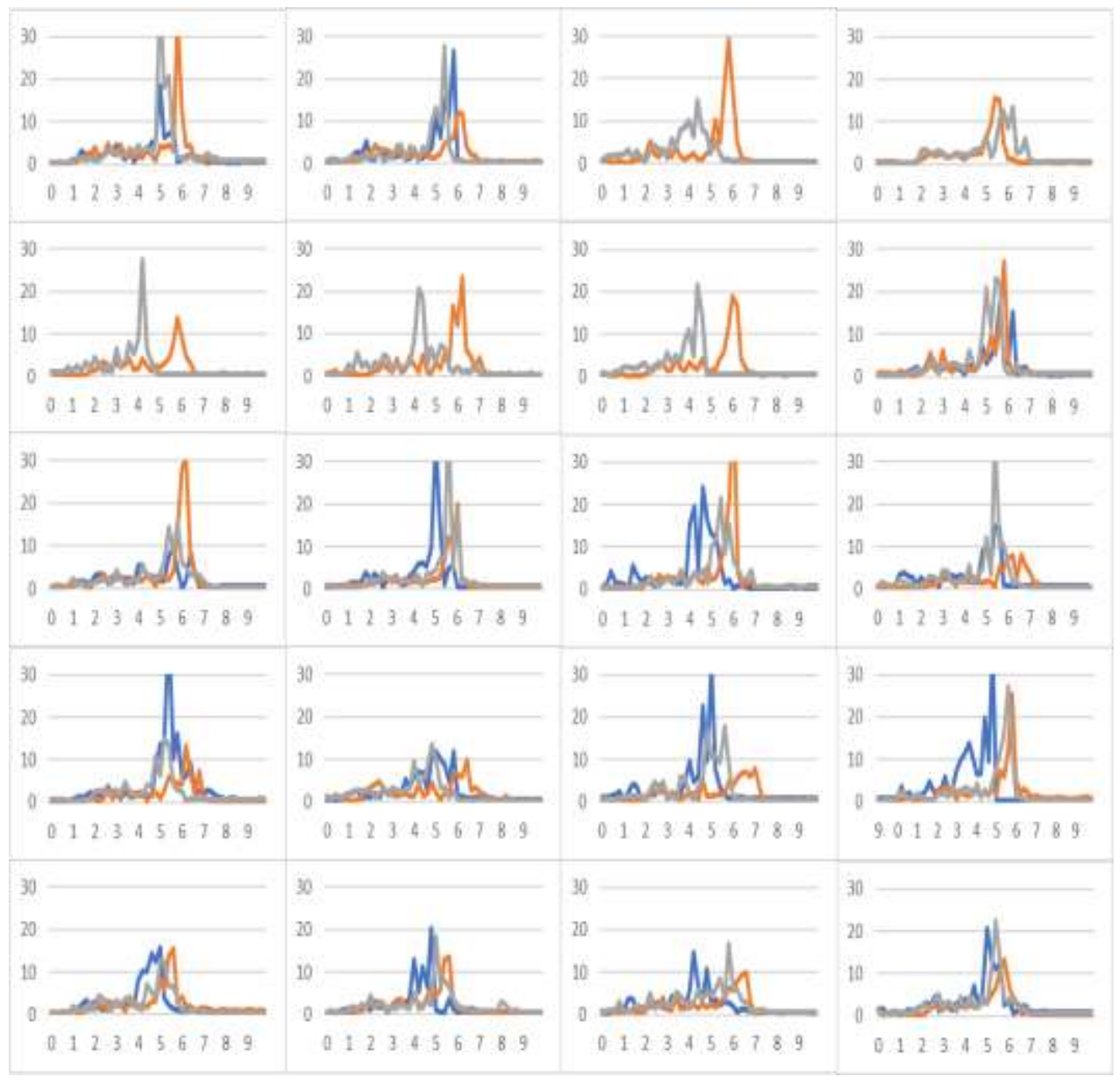

Figure 5. Experimental Results of Three Fixed Posture Experiments $x$-axis: experimental time (sec.), y-axis: geometric mean of triaxial acceleration $\left(\mathrm{m} / \mathrm{s}^{2}\right)$ 
Table 3. Result of Event Generation According to Application of Proposed Algorithm

\begin{tabular}{|c|c|c|c|c|c|c|c|c|c|c|}
\hline \multirow{2}{*}{$\begin{array}{l}\begin{array}{r}\text { Threshold } \\
\left(\mathrm{m} / \mathrm{s}^{2}\right)\end{array} \\
\text { Threshold } \\
\left(\mathrm{m} / \mathrm{s}^{2}\right)\end{array}$} & \multicolumn{2}{|c|}{1.6} & \multicolumn{2}{|c|}{1.4} & \multicolumn{2}{|c|}{1.2} & \multicolumn{2}{|c|}{1.0} & \multicolumn{2}{|c|}{0.8} \\
\hline & $\begin{array}{l}\text { Sig. } \\
\text { Fall }\end{array}$ & Fall & $\begin{array}{l}\text { Sig. } \\
\text { Fall }\end{array}$ & Fall & $\begin{array}{l}\text { Sig. } \\
\text { Fall }\end{array}$ & Fall & $\begin{array}{l}\text { Sig. } \\
\text { Fall }\end{array}$ & Fall & $\begin{array}{l}\text { Sig. } \\
\text { Fall }\end{array}$ & Fall \\
\hline 10.0 & 81.7 & 84.2 & 81.7 & 84.2 & 80 & 84.2 & 79.2 & 84.2 & 68.3 & 84.2 \\
\hline 9.8 & 85 & 87.5 & 85 & 87.5 & 83.3 & 87.5 & 82.5 & 87.5 & 71.7 & 87.5 \\
\hline 9.6 & 86.7 & 89.2 & 86.7 & 89.2 & 85 & 89.2 & 84.2 & 89.2 & 72.5 & 89.2 \\
\hline 9.4 & 89.2 & 91.7 & 89.2 & 91.7 & 87.5 & 91.7 & 86.7 & 91.7 & 75 & 91.7 \\
\hline 9.2 & 89.2 & 91.7 & 89.2 & 91.7 & 87.5 & 91.7 & 86.7 & 91.7 & 75 & 91.7 \\
\hline 9.0 & 89.2 & 91.7 & 89.2 & 91.7 & 87.5 & 91.7 & 86.7 & 91.7 & 75 & 91.7 \\
\hline 8.8 & 89.2 & 91.7 & 89.2 & 91.7 & 87.5 & 91.7 & 86.7 & 91.7 & 75 & 91.7 \\
\hline 8.6 & 89.2 & 91.7 & 89.2 & 91.7 & 87.5 & 91.7 & 86.7 & 91.7 & 75 & 91.7 \\
\hline 8.4 & 90.8 & 93.3 & 90.8 & 93.3 & 89.2 & 93.3 & 88.3 & 93.3 & 76.7 & 93.3 \\
\hline 8.2 & 90.8 & 93.3 & 90.8 & 93.3 & 89.2 & 93.3 & 88.3 & 93.3 & 76.7 & 93.3 \\
\hline 8.0 & 95 & 97.5 & 95 & 97.5 & 93.3 & 97.5 & 92.5 & 97.5 & 80.8 & 97.5 \\
\hline 7.8 & 95 & 97.5 & 95 & 97.5 & 93.3 & 97.5 & 92.5 & 97.5 & 80.8 & 97.5 \\
\hline 7.6 & 95 & 97.5 & 95 & 97.5 & 93.3 & 97.5 & 92.5 & 97.5 & 80.8 & 97.5 \\
\hline 7.4 & 95.8 & 97.5 & 95.8 & 97.5 & 93.3 & 97.5 & 92.5 & 97.5 & 80.8 & 97.5 \\
\hline 7.2 & 95.8 & 97.5 & 95.8 & 97.5 & 93.3 & 97.5 & 92.5 & 97.5 & 80.8 & 97.5 \\
\hline 7.0 & 95.8 & 97.5 & 95.8 & 97.5 & 93.3 & 97.5 & 92.5 & 97.5 & 80.8 & 97.5 \\
\hline 6.8 & 96.7 & 98.3 & 96.7 & 98.3 & 94.2 & 98.3 & 93.3 & 98.3 & 81.7 & 98.3 \\
\hline 6.6 & 96.7 & 98.3 & 96.7 & 98.3 & 94.2 & 98.3 & 93.3 & 98.3 & 81.7 & 98.3 \\
\hline
\end{tabular}

The value of the acceleration varied from $10 \mathrm{~m} / \mathrm{s}^{2}$ to $40 \mathrm{~m} / \mathrm{s}^{2}$ depending on the physique of the experimenter and the behavior of the experimenter when he fell. After all the movements, such as falling and rolling, the accelerations were kept below $1 \mathrm{~m} / \mathrm{s}^{2}$ when the experimenter took no motion. This value showed a big difference compared to walking and falling.

Figure 5 shows the result of the fixed posture experiment of three subjects. The $\mathrm{x}$-axis is the time after the start of the experiment and is in seconds. The y-axis is the geometric mean of the three-axis acceleration values of the experimenter, and the unit is $\mathrm{m} / \mathrm{s}^{2}$. It is the moment when the experimenter collapses 3 seconds after the start of the experiment, and the acceleration is noticeably increased at this time.

Based on the experimental data, we tested the algorithm as follows to check whether the proposed algorithm is meaningful and whether there is a specific threshold value that can accurately generate a falling event.

Table 3 shows the result obtained by changing the threshold value of the algorithm to the current experimental data. The threshold $f$ value is used to find the Fall event, and the unit is $\mathrm{m} / \mathrm{s}^{2}$, from 10.0 to 6.6 , with a drop of 0.2 . The threshold $_{s f}$ value is used to find the Significant Fall event after the collapse, and the unit is $\mathrm{m} / \mathrm{s}^{2}$, which is reduced from 0.2 to 1.6 by 0.2 . 
Looking at the change in the threshold $_{f}$ value, the accuracy increases greatly when the value is less than 9.4, and there is a small change thereafter. Since the positive error is mostly zero, but there is a positive error of $1.7 \%$ when the value is less than 7.4 , the final threshold $_{f}$ value should be determined considering the correlation between positive and negative errors.

In the case of the threshold $_{s f}$ values of 1.6 and 1.4, the threshold value of Significant Fall was $97.6 \%$ of the total fall. However, the value of Significant Fall was decreased from 1.0, which is 94.7 to 0.8 , which is $82.3 \%$, respectively. Based on the same threshold $_{s f}$ value, a gradual decline to 1.0 and a sharp decline at 0.8 .

In this experiment, it is confirmed that there is a threshold value that can expect more than certain level of accuracy while considering the correlation between negative and positive errors of Fall event and Significant Fall event.

\section{Conclusion}

In order to meet the requirements of the convergence security service, our proposed platform relies on multiple sensors. Its goal is to track user locations and to monitor access to restricted facilities. To achieve this goal, we designed and implemented a communication protocol between the device and the relay driver and a database for storing the sensed data. In addition, we propose a hazard event generation algorithm to detect and to generate the user hazard events based on the sensed data.

However, we still have not completed target tracking technology based on indoor / outdoor positioning. We intend to improve the completeness of intelligent convergence security platform by carrying out these studies in the future.

\section{Acknowledgments}

This research was supported by the MISP (Ministry of Science, ICT \& Future Planning), Korea, under the National Program for Excellence in SW) (2016-0-00017) supervised by the IITP(Institute for Information \& communications Technology Promotion).

\section{References}

[1] K. H. Park, I. K. Ra, and C. S. Kim, "Study on the Vulnerability Level of Physical Security and Application of the IP-Based Devices", International Journal of Smart Home, vol. 9, no. 10, (2015), pp. 63-68.

[2] K. Kang and J. Kim, "A Case Study on Converged Security with Event Correlation of Physical and Information Security", International Journal of Security and Its Applications, vol. 9, no. 9, pp. 77-94.

[3] L. Q. Xu, J. L. Landabaso and B. Lei, "Segmentation and tracking of multiple moving objects for intelligent video analysis", BT technology Journal, vol. 22, no. 3, (2004), pp. 140-150.

[4] A. Costin, "Security of CCTV and Video Surveillance Systems: Threats, Vulnerabilities, Attacks, and Mitigations", In Proceedings of the 6th ACM International Workshop on Trustworthy Embedded Devices, (2016).

[5] B. Madhuravani and M. Rashmika, "Motion sensor and face recognition based surveillance system Using Raspberry Pi”, International Journal of Advanced Research in Computer Science, vol. 5, no. 8, (2017).

[6] K. J. Woo, "Resaerch Trend and Cocentualization of Defense Industry Security from Convergence Security Perspective", Journal of Information and Security, vol. 15, no. 6, (2015), pp. 69-78.

[7] D. Lee and O. H. Ha, "A study on Improved Convergence Security Monitoring System model", Journal of Information and Security, Vol. 11, No. 5, pp. 3-12. 2011.

[8] H. K. Ahn, "A Study on the Development of Convergence Security with the Changes in Security Environments", Journal of Information and Security, vol. 11, no. 5, (2011), pp. 31-40.

[9] M. Yim, "Development of Measures of Information Security Policy Effectiveness to Maximize the Convergence Security", Journal of the Korea Convergence Society, vol. 5, no. 4, (2014), pp. 27-32.

[10] I. Ha, "Security and Usability Improvement on a Digital Door Lock System based on Internet of Things", International Journal of Security and Its Applications, vol. 9, no. 8, (2015), pp. 45-54. 


\section{Authors}

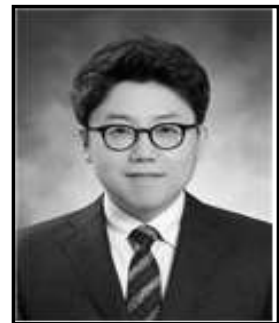

Junho Jeong, received the B.S. degree from the Dept. of Computer Science, Dongguk University, Seoul, Korea, in 2007, and M.S. and Ph.D. degrees from the Dept. of Computer Engineering, Dongguk University, Seoul, Korea in 2009 and 2015, respectively. Currently, he is a research professor of the Electronic Commerce Institute, Dongguk University, Gyeongju, Korea. His research areas include Privacy Preserving, Distributed System, Network Security and Secure Software.

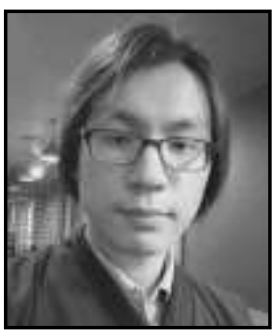

Dong Ha Park, is an undergraduate student from Dept of Computer Science and Engineering, Dongguk University, Seoul, Korea since 2012. His interest is concurrency and network programming. He performed research on parallel processing and performance comparison between programming languages.

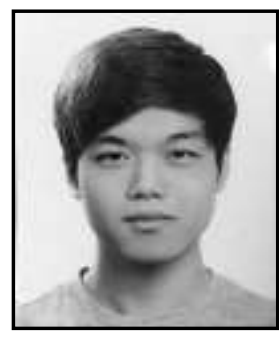

Jun Young Lee, is an undergraduate student from the Dept. of Computer Science, Dongguk University, Seoul, Korea. He is interested in Secure Software, Block Chain Network and Web Programming.

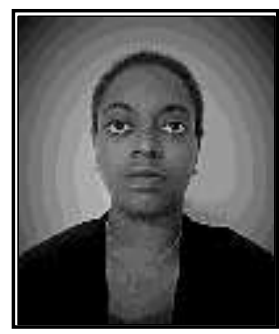

Uduakobong George Offong, received a B.S degree from the Dept. of Mathematics and Physics, College of the Ozarks, Missouri, USA, in 2008. She is currently on an M.S degree in Information Security from the Dept of Computer Engineering, Dongguk University, Seoul, Korea.

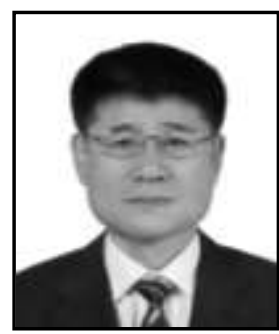

Seman Oh, received the B.S. degree from the Seoul National University, Seoul, Korea, in 1977, and M.S. and Ph.D. degrees from the Dept. of Computer Science, Korea Advanced Institute of Science and Technology, Seoul, Korea in 1979 and 1985, respectively. He was a Dean of the Dept. of Computer Science and Engineering, Graduate School, Dongguk University from 1993-1999, a Director of SIGPL in Korea Institute of Information Scientists and Engineers from 2001-2003, a Director of SIGGAME in Korea Information Processing Society from 2004-2005. Currently, he is a Professor of the Dept. of Computer Science and Engineering, Dongguk University, Seoul, Korea. His research areas include smart system solutions, programming languages, and embedded systems. 


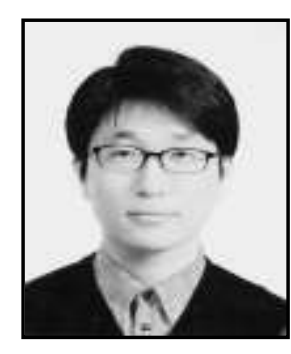

Yunsik Son, received the B.S. degree from the Dept. of Computer Science and Engineering, Dongguk University, Seoul, Korea, in 2004, and M.S. and Ph.D. degrees from the Dept. of Computer Science and Engineering, Dongguk University, Seoul, Korea in 2006 and 2009, respectively. He was a research professor of Det. of Brain and Cognitive Engineering, Korea University, Seoul, Korea from 2015-2017. Currently, he is an Assistant Professor of Dept. of Computer Science and Engineering, Dongguk University, Seoul, Korea. His research areas include secure software, programming languages, compiler construction, mobile/embedded systems, and uHealthcare. 